\title{
RELATIONSHIP BETWEEN THE SELF-SUSTAINED ACTIVITY OF THE CEREBRAL CORTEX AND THE EFFERENT DISCHARGE OF CARDIAC SYMPATHETIC AND PARASYMPATHETIC FIBERS
}

\author{
J. Alanis, D. Mascher And J. Miyamoto* \\ Department of Physiology, Instituto Nacional de Cardiologia, México
}

Several authors, working under different experimental conditions (BOCHEFontAine, 1875 ; Dusser de BARENne, 1924 ; Hoff, 1936, 1940, 1951 ; Crouch, 1936; KAAdA, 1949; Delgado, 1960 ; etc.) have indicated that the cerebral cortex may exert an influence on the cardiovascular system. In a previous paper, AlANis et al. (1965) suggested that some of the neurovegetative changes induced by cortical activity were mediated by the sympathetic system. The aim of the present study was to evaluate the magnitude and temporal relationships between sigmoid cortical activity and the efferent discharges of vagal and sympathetic fibers.

\section{METHODS}

The experiments were performed on mongrel dogs from 10 to $22 \mathrm{~kg}$ of body weight. Morphine was injected $(0.01 \mathrm{~g})$ and the surgical procedures were performed under ether anaesthesia. In order to keep a constant respiratory volume a pump was connected through a tracheal cannula and galamine triethiodide was injected (Flaxedil "Specia"). The ether anaesthesia was discontinued when the animals were paralyzed. The thorax was opened by resection of the second and third left ribs. The postganglionic left sympathetic fibers (inferior cardiac nerve) were dissected near the inferior cervical sympathetic ganglion. The left vagal trunk was isolated and desheathed near the heart in order to identify some of the cardiac fibers. The identification of sympathetic and parasympathetic fibers was achieved by the electrical stimulation of fine strands which produced either an increase or decrease in heart rate. After this procedure the nerve trunks were severed. The central cut end of both nerves, vagal and sympathetic, were placed on platinum recording electrodes immersed in a mineral oil pool. Action potentials from nerve fibers were recorded by a dual beam oscilloscope (Tektronix 502). The quantification of nerves electrical activity was achieved

Received for publication October 16, 1965

宮本ジュリオ

* Present address, Osaka City University, Medical School, Japan. 
by summing up the frequency and amplitude of response with a 5P3 Grass integrator unit. The measurements of the areas under the integrated curves are proportional to the electrical energy of nerve or neurone activity. The animals were placed on a stereotaxic apparatus (Horsley-Clarke type) and cranial trepanations were performed in order to expose the left sigmoid cerebral cortex convolutions and some other cerebral regions. Electrodes for stimulation and recording were introduced (1 to 1.5 $\mathrm{mm}$ depth) into the different cortical regions. The electrodes were a pair of platinum needles (200 microns tip diameter) separated 200 microns from each other. The exposed areas were sealed with mineral oil. The same pair of electrodes served for recording and stimulation. The self-sustained activity of the cerebral cortex (toniccolonic type) was evoked by electrical stimulation $(0.5-2.0 \mathrm{~mA})$. The stimuli were trains of square pulses of $1.5 \mathrm{msec}$ duration and a frequency of 20 to 30 per sec. The stimulation period varied from 4 to 8 seconds. The recording electrodes were connected to EMG preamplifiers (Grass $5 \mathrm{P} 1$ ) and in some cases the activity was integrated (5P3 Grass) and recorded in the same ink writing apparatus (Grass Poligraph). The systemic blood pressure was measured by means of a transducer (Statham P23 Dc) calibrated with a mercury manometer.

In some experiments a reservoir containing heparinized blood and a large volume of air at a given pressure was connected through large tubes to both common carotid and femoral arteries. This set-up maintained the blood pressure constant at a given level. With this compensator system it was possible to keep the blood pressure constant, within $\pm 10 \mathrm{mmHg}$ even in the experiments in which otherwise a considerable rise in blood pressure would have occurred.

The pupil size was continuously recorded using a circuit, which changed the input voltage to a DC preamplifier according to the modifications in the pupillary diameter estimated by an observer.

\section{RESULTS}

Changes in the discharge patterns of the sympathetic cardiac nerves produced by the cerebral self-sustained activity. The electrical activity of sympathetic postganglionic fibers from both the superior cervical and the "stellate" ganglia increased when the anterior or posterior sigmoid gyri were stimulated. If the electrical stimulation was strong enough to produce a self-sustained activity in these cortical areas, the increase in sympathetic activity was greater than during the stimulation period. The augmented sympathetic activity lasted as long as the cerebral self-sustained episode.

The sympathetic activity augmented either by an increase in the frequency of response and/or the amplitude of the responses (FIGs. $1 \mathrm{~B}$ and 2B). In some experiments a rhythmic sympathetic discharge was transformed into a continuous activity (FIGS. 3B, 4B, and 5B). In others the rhythmic activity persisted but with a greater magnitude (FIgs. 1B and 2B). The magnitude of the increment in sympathetic activity, observed during the self-sustained activity was not related to the frequency of the electrical stimuli or to the length of the stimulation period. When the threshold for the self-sustained activity was reached, the cortical response appeared and its characteristics determined the magnitude of increase in sympathetic discharge (FIGs. 1B, C 

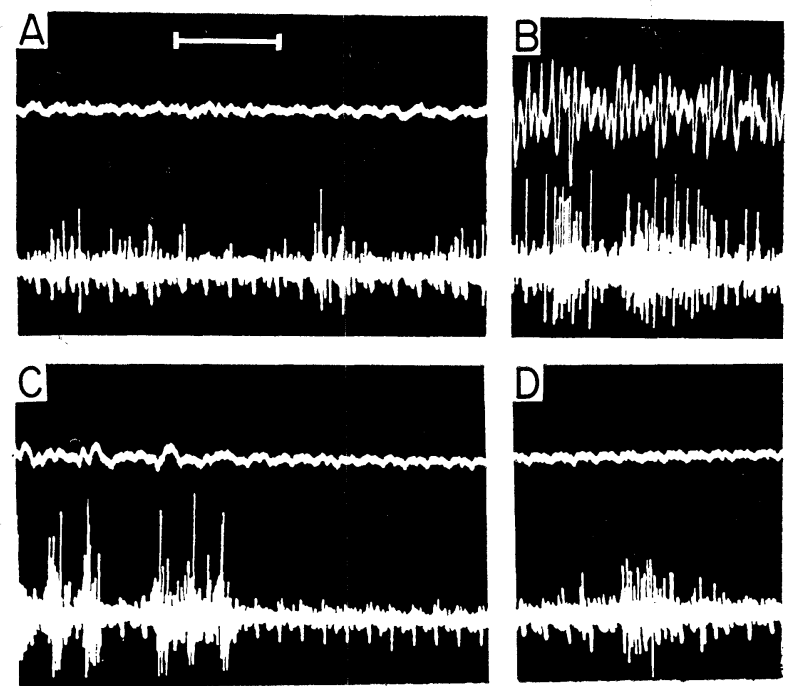

FIG. 1. Increase in sympathetic activity caused by the cortical self-sustained activity. Upper trace, recording from the anterior sigmoid gyrus. Lower trace, action potentials (75-150 microvolts) from a fine strand of the post ganglionic fibers of inferior cardiac nerve. A, control. B, initial part of a self-sustained activity episode produced by electrical stimulation $(20 / \mathrm{sec})$ and the corresponding increase in sympathetic activity. C, final part of the self-sustained cortical episode which lasted 84 seconds. Note that simultaneously with the cessation of the cortical response the sympathetic discharge also disappeared. In this case the sympathetic activity was practically abolished and reappeared after 4 sec. in D. Calibration $1 \mathrm{sec}$.

and $2 \mathrm{~B}, \mathrm{C})$. This increase was, in general, parallel to the magnitude of cortical self-sustained activity recorded from the sigmoid gyri. The analysis of the integrated responses confirmed this statement (FIG. 6). In a few experiments the above mentioned relationship was observed only at the beginning of the self-sustained activity.

The self-sustained cortical response, evoked by the stimulation of convolutions others than the sigmoid, did not produce by itself changes in the sympathetic activity. An example of this is illustrated in FIG. 8 . In this case the posterior portion of the left ectolateralis gyrus was stimulated and a self-sustained response was evoked in this area. In spite of the long duration $(39 \mathrm{see})$ of this self-sustained activity there were no modifications either in the sympathetic action potentials, in the systemic blood pressure, in the heart rate or in the pupillary size. Such modification appeared only when the activity spread from the ectolateralis to the sigmoid gyri (FIG. 8C). Similar results were obtained when stimulating the following cerebral regions: lateralis and suprasylvius gyri, middle portion of cingulate convolution, anterior portion of the cerebellar lobule and posterior part of the hypothalamus. 


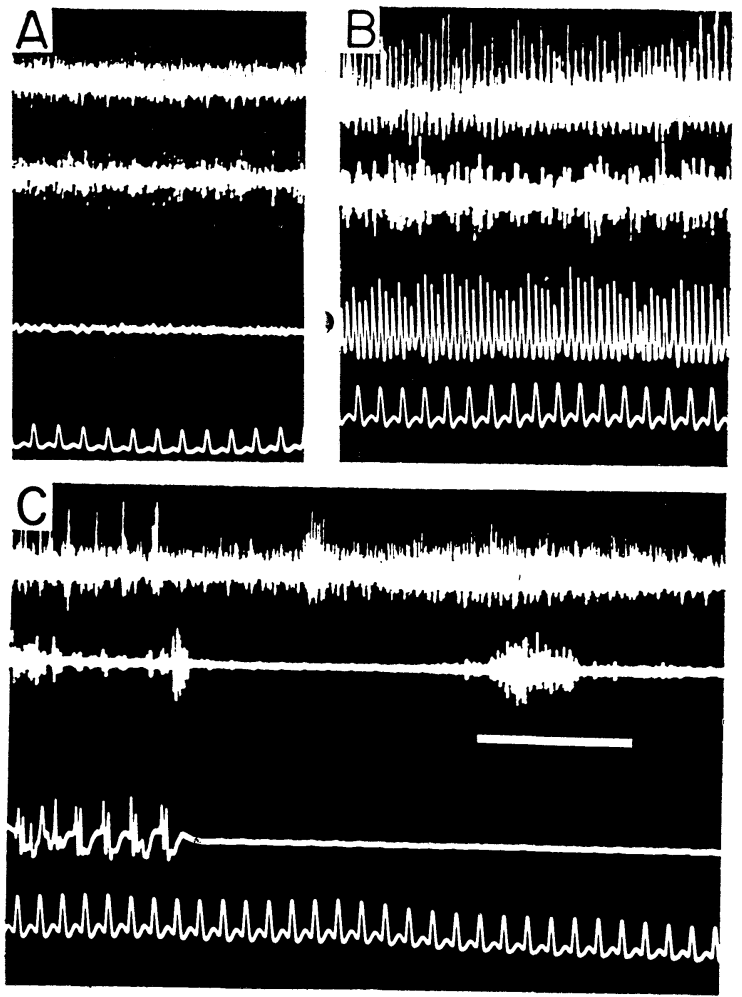

FIG. 2. Changes in parasympathetic and sympathetic activities produced by the self-sustained cortical response from sigmoid gyri. The records from the top downwards correspond respectively to the electrical activity of parasympathetic cardiac fibers, to inferior cardiac nerve, to the cortical response from the sigmoid gyrus and to the systemic blood pressure. A, control. Femoral artery blood pressure $(120 \mathrm{mmHg})$. Heart rate $(3.6 / \mathrm{sec})$. B, during a cortical self-sustained episode which lasted $72 \mathrm{sec}$. Note the increment in blood pressure $(180 \mathrm{mmHg})$ and in heart rate $(4.0 / \mathrm{sec})$. The modification in heart rate was small due to the simultaneous increase in vagal and sympathetic discharges. $C$, during the abrupt ending of self-sustained cortical activity. Both vagal and sympathetic activities ceased abruptly and blood pressure fell simultaneously with the inhibition of cortical response. See that vagal discharge reappeared with a greater magnitude during the marked sympathetic inhibition, which only lasted $4 \mathrm{sec}$. Sympathetic nerves discharged again while the cortical activity still was inhibited. Calibration $2 \mathrm{sec}$.

The self-sustained activity originated in any of these areas did not produce by itself an increase in sympathetic activity.

Two main stages have been described for the self-sustained cortical activity (Rosenblueth et al., 1942). A tonic stage characterized by a high frequency response and a second stage by a synchronized low frequency activity which produces motor activation of the clonic type. The presence 


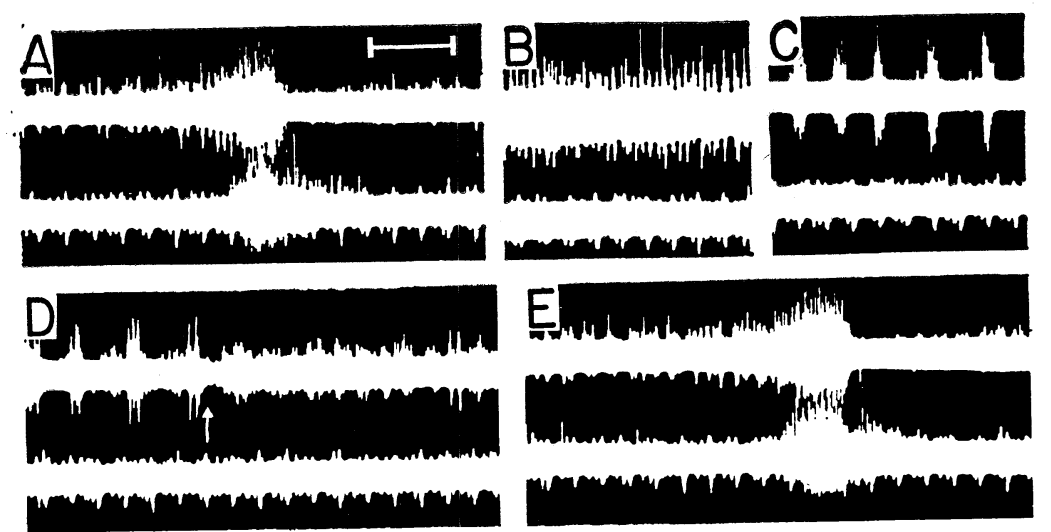

FIG. 3. Effects produced by the "tonic-clonic" cortical stages. A, control. In this experiment sympathetic (upper record) and vagal discharges (lower record) occurred almost simultaneously. B, immediately after the stimulation period of the anterior sigmoid gyrus and during the tonic stage of a self-sustained cortical response. Sympathetic activity was increased while vagal response was inhibited. $C$, synchronization of sympathetic discharge with the cortical clonic activity (83 sec after B). D, at the arrow the self-sustained cortical episode ceased and the sympathetic discharge ended, remaining diminished for approximately 300 sec. until it reached the control pattern in E. Note that the vagal response was inhibited all the time and the control discharge reappeared (E) simultaneously with the sympathetic activity. Calibration $1 \mathrm{sec}$.

of these two stages suggested the term of "cerebral tonic-clonic activity" which described the type of motor responses provoked by this cortical activity. The present results indicate that the sympathetic activity of cardiac nerves also present the two types of responses (tonic and clonic) which coincided with the cortical stages (FIGs. 3B, $4 \mathrm{~B}$ and $5 \mathrm{~B}$ ). During the clonic period the sympathetic discharges were synchronized and grouped in volleys which had the frequency of the clonic cerebral activity (FIGS. 3C, D and 5C, D, E).

The duration of the self-sustained cortical activity fluctuated from 50 to 300 seconds. In general, one third of this time corresponded to the tonic stage and the other two thirds to the clonic period. A similar temporal course was followed by the sympathetic discharges.

The self-sustained activity usually ended suddenly and it was followed by a considerable reduction in the basal cortical electrograms. This reduction lasted several seconds and when the activity reappeared it had similar magnitude and characteristics as before the initiation of the self-sustained activity. The magnitude of sympathetic discharge decreased when the selfsustained response suddenly ceased. The sympathetic activity decreased to such an extent that the recorded potentials were smaller than those observed before the self-sustained cortical activity (FIG. 1C). This reduction in sympathetic activity lasted 2 to 13 seconds and in a few experiments the complete 


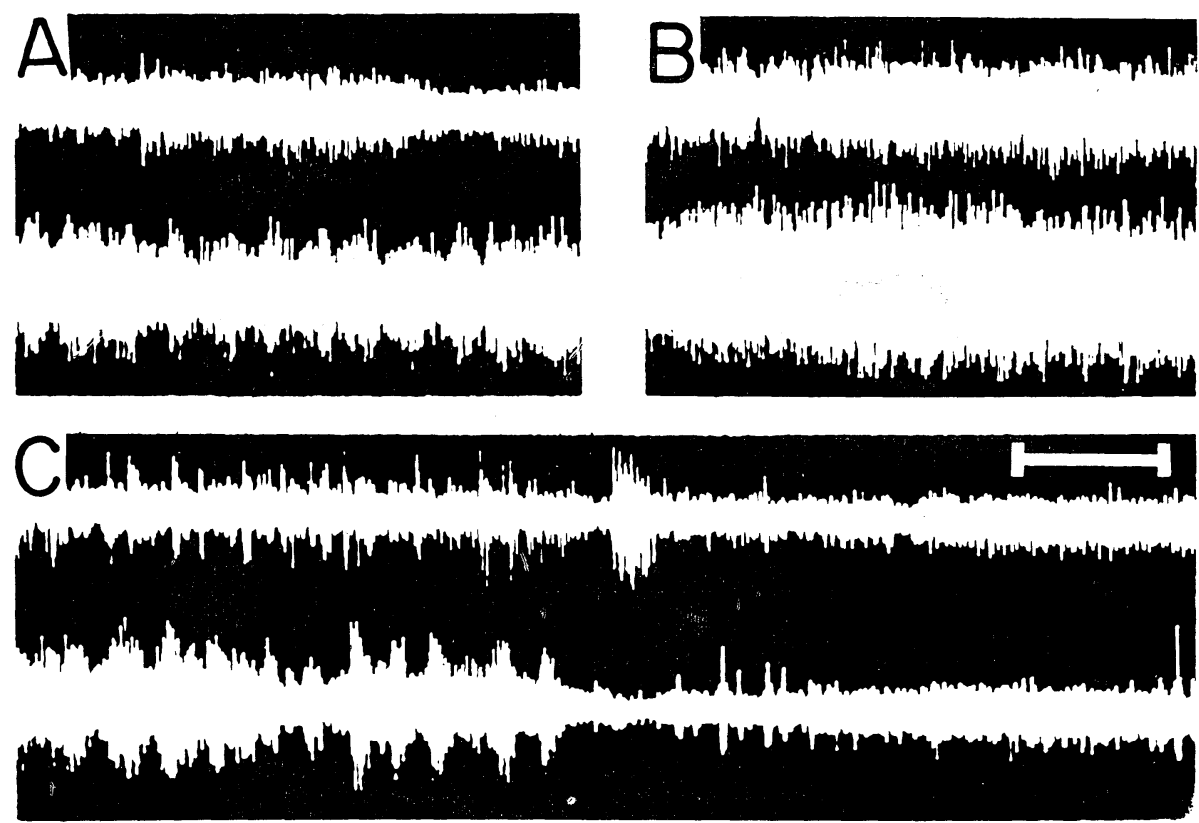

FIG. 4. Reciprocity between vagal (upper trace) and sympathetic (lower trace) activities. A, control. B, increase in both vagal and sympathetic discharges during a tonic cortical self-sustained activity episode evoked in the anterior sigmoid gyrus. C, recordings taken $72 \mathrm{sec}$ after $\mathrm{B}$. Note that the sudden reduction in sympathetic action potentials occurred simultaneously with a burst-like discharge in vagal fibers. These two events coincided with the abrupt cessation of sigmoid gyrus self-sustained activity. Calibration $1 \mathrm{sec}$.

recovery was seen only after 200-300 seconds (FIG. 3E). In other cases the activity of the sympathetic nerves was practically abolished (FIG. 2C). When the sympathetic activity reappeared it had similar characteristics to those of the control pattern (FIGs. 3E and 5E). At this moment there was no longer a relationship with cortical activity, since this could still be inhibited while the sympathetic discharge was already growing (FIGS. 1D and 2C).

In some experiments, the self-sustained activity was interrupted temporarily. This interruption had a short duration and was followed by the renewal of the same type of activity. The sympathetic action potentials also disappeared abruptly during this cessation of cortical response and reappeared simultaneously with it (FIG. 7).

The increase in the magnitude of sympathetic activity produced by the self-sustained cortical activity appeared independently of the values in systemic blood pressure. This was also true when the important changes in blood pressure were minimized or dampened at values of $100-120 \mathrm{mmHg}$. This was accomplished by means of a sympathetic blocking agent $(\mathrm{AM} / 277$, Miles 


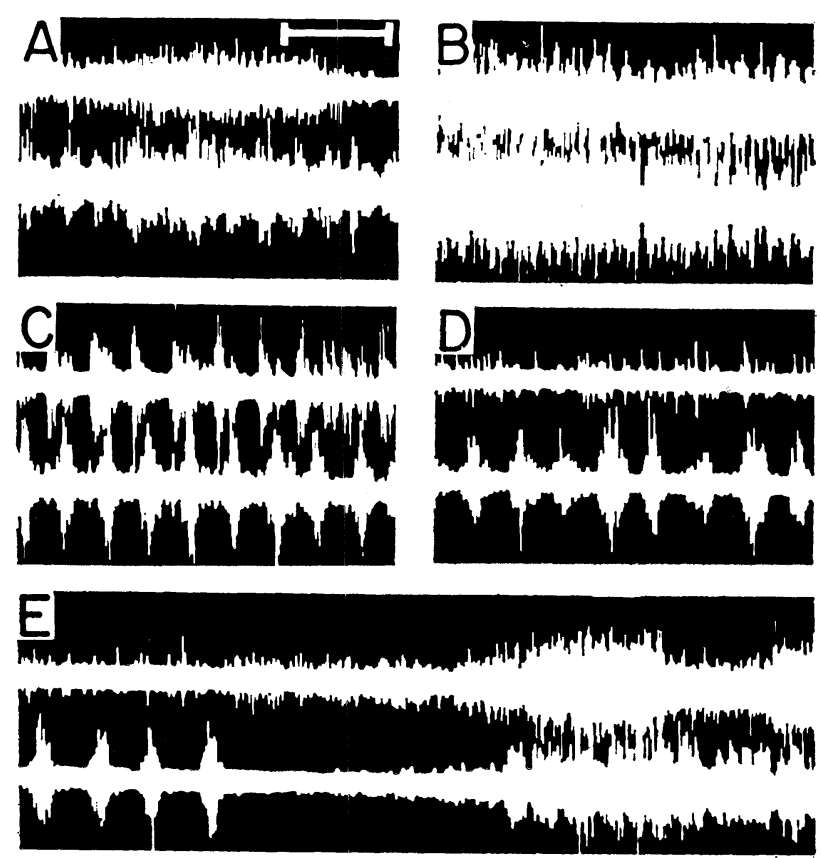

FIG. 5. Different patterns of vagal discharge occurring during a self-sustained cortical activity evoked in the sigmoid gyri. A, control. Action potentials from parasympathetic cardiac fibers (upper record) and sympathetic post ganglionic fibers (lower record). B, sixteen seconds after the stimulation period and during the tonic stage of cortical activity. C, during the clonic stage both vagal and sympathetic discharges are synchronized with the cortical responses. Records taken $33 \mathrm{sec}$ after B. D, ( $81 \mathrm{sec}$ after C) the vagal discharge is inhibited while sympathetic fibers still respond synchronically with the cortex. E, the last sympathetic volley (lower record) corresponds to the abrupt cessation of the selfsustained cortical activity. Sympathetic activity reappeared with characteristics similar to those of control, in spite of the marked cortical inhibition, and vagal discharge increased again at the time the sympathetic activity reappeared. Records in $\mathrm{E}$ taken $8 \mathrm{sec}$ after D. Calibration, $1 \mathrm{sec}$.

Lab.) or by the compensating system. The reduction or abolition of sympathetic discharge, concomitant with the cessation of the self-sustained cortical response, was also independent of the blood pressure values. Neither the augmentation nor the abolition of sympathetic discharge were changed after bilateral cervical vagotomy.

The above mentioned modifications in sympathetic activity were observed in the left inferior cardiac nerve when the ipsilateral sigmoid gyrus was stimulated, but similar modifications were observed when the contralateral convolutions were stimulated (right sigmoid gyri). It was difficult to circumscribe the self-sustained activity to the stimulated area since the cortical 


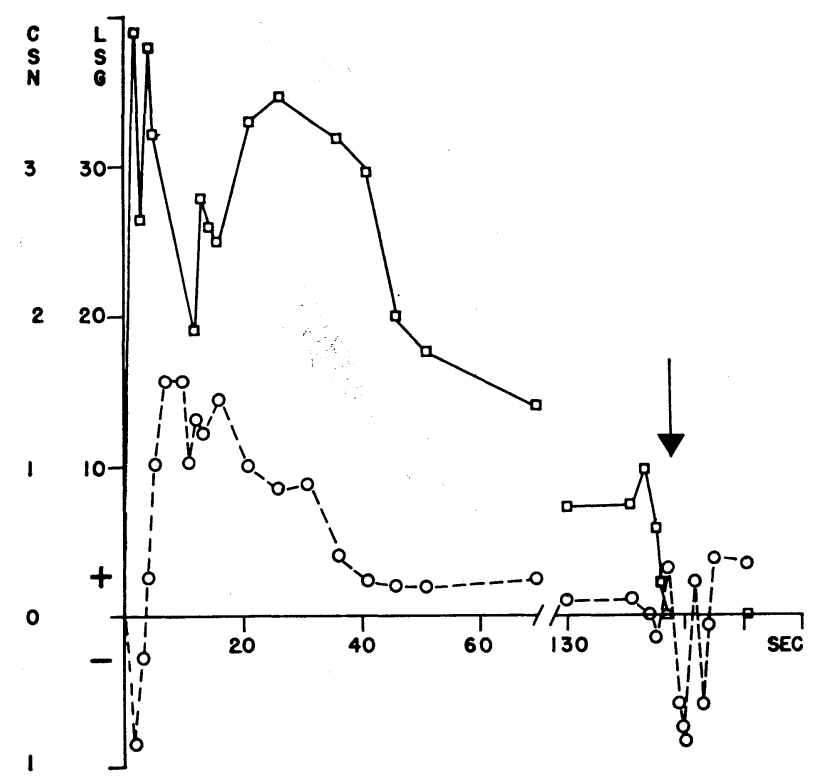

FIG. 6. Relationship between the magnitude of the changes in self-sustained cortical activity of the anterior sigmoid gyrus $(\square)$ and the sympathetic activity of inferior cardiac nerve (O). Ordinates, left scale, changes in sympathetic cardiac nerve activity (CSN) expressed in times fold; right scale left sigmoid gyrus activity (LSG) in times fold of increase. Abscissae, time in sec after the cessation of the stimulation of the sigmoid gyri. The evaluation of both cortical and sympathetic activities was performed using a Grass 5P3 preamplifier and integrator (see FIG. 9). Note that the changes in the magnitude of responses are parallel. At the arrow the self-sustained cortical activity ceased abruptly and the sympathetic discharge was inhibited.

response usually spread to the contralateral homologous gyrus.

The records illustrated in this paper correspond to the activity of the inferior cardiac nerve, but similar changes occurred in other cervical sympathetic fibers.

Other effects elicited by the self-sustained cortical activity which occurred simultaneously with the modifications in sympathetic response. At the onset of a period of self-sustained cortical activity the heart rate, systemic blood pressure and pupillary size increased (FIGS. 2B and 8). The magnitude and temporal course of these increments kept a close relationship with the magnitude of the self-sustained cortical response. In some experiments the increase in heart rate lasted as long as the self-sustained cortical activity (FIGs. 8D) but in others, the increase appeared only during the initial part of the cortical response. In the latter case the heart rate returned to the control values while the self-sustained cortical activity was still present. When the self- 

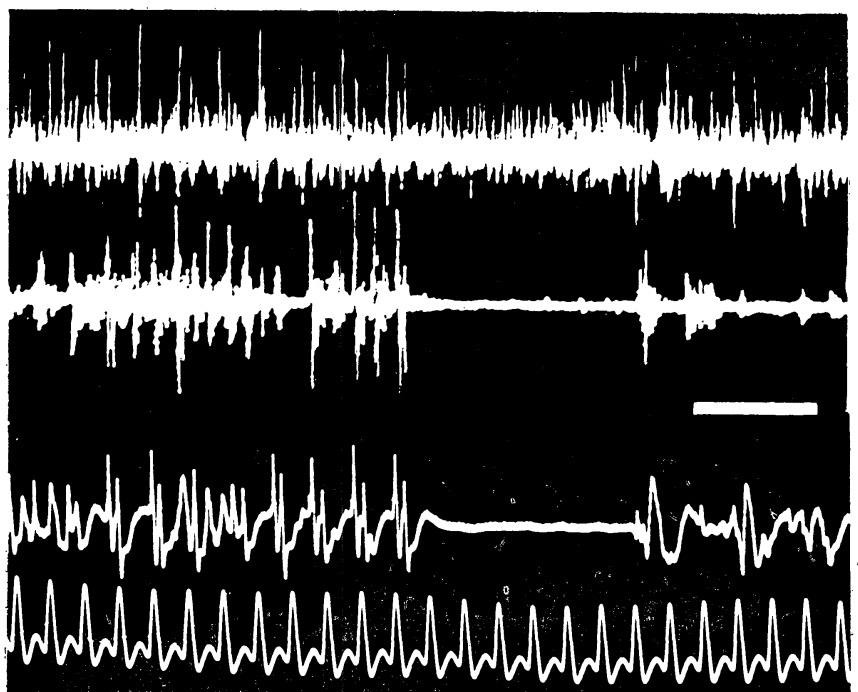

FIG. 7. Short spontaneous cessation of the cortical self-sustained response which was accompanied by a sudden abolition of sympathetic activity and a diminution in vagal discharge. The traces from the top downwards correspond respectively to vagal fibers action potentials, sympathetic activity, sigmoid gyrus response and systemic blood pressure. When the cortical activity ceased the sympathetic discharge was practically abolished and vagal activity decreased. When the cortical silent period encled the activities of both nerves appeared again. Calibration, 1 sec.

sustained cortical response ended abruptly the heart rate diminished also in an abrupt manner (FIGS. 8D, 9 and 10). The cardiac frequency reached lower values than those recorded before the stimulation period (FIGS. 8D and 10) and occurred concomitantly with inhibition of the sympathetic activity already described (FIGs. 9 and 10). The duration of this bradycardia was similar to that of the reduction in sympathetic activity $(2$ to $13 \mathrm{sec}$ ). The bradycardia occurred when the vagi nerves were intact. After cervical vagotomy the reduction in heart rate was slight or did not appear. The bradycardia did not appear when the changes in systemic blood pressure were minimized by means of the different procedures already mentioned.

Changes in the pattern of discharge of vagal cardiac nerves produced by the cerebral self-sustained cortical activity. The electrical activity of parasympathetic cardiac fibers was substantially modified during the self-sustained response produced by the stimulation of sigmoid gyrus. Although in general, an increase in vagal discharge was evoked during the tonic-clonic cortical activity, the changes can be classified in four groups. 1) An increase in the amplitude and frequency of vagal fiber discharge, which occurred at the onset of the self-sustained cortical response (FIG. 2). There was a direct relation- 


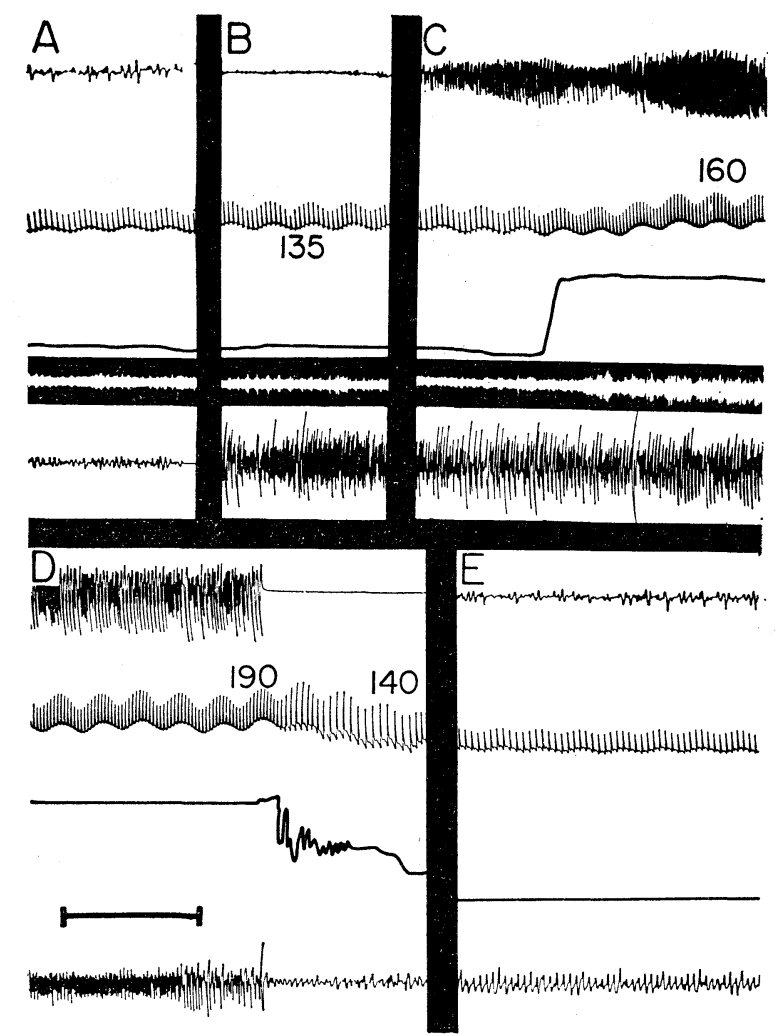

FIG. 8. The activation of the anterior sigmoid gyrus, produced by self-sustained activity of the ectolateralis convolution. The traces from the top downwards correspond respectively to the electrogram of the anterior sigmoid gyrus, to femoral artery blood pressure $(\mathrm{mmHg})$, to the pupillary size, to the electrical activity of the left inferior cardiac nerve and to the ectolateralis convolution's electrogram. A, control. B, after the electrical stimulation of the ectolateralis convolution, which produced in this area a self-sustained activity. Although this activity had a great amplitude, frequency and duration, it did not produce any changes in the autonomic effectors under study. Only when the self-sustained activity from the ectolateralis convolution spread to the anterior sigmoid gyrus (C, $39 \mathrm{sec}$ after B) and when it reached certain magnitude, an increase in the sympathetic cardiac fibers activity, blood pressure, heart rate and pupillary size were observed. Note that the first change was the pupils' dilatation and when the sympathetic activity increased, both the heart rate and blood pressure started to augment. D, the increases in blood pressure, heart rate and pupillary size lasted until the self-sustained activity of the anterior sigmoid gyrus ceased abruptly. During the cortical silent period the heart rate decreased considerably (bradycardia) and the blood pressure and pupillary size returned to the control values. See that the fall in blood pressure and the changes in heart rate occurred also abruptly and simultaneously with cessation of the cortical activity. E, recovery $15 \mathrm{sec}$ after D. Calibration, $10 \mathrm{sec}$. 


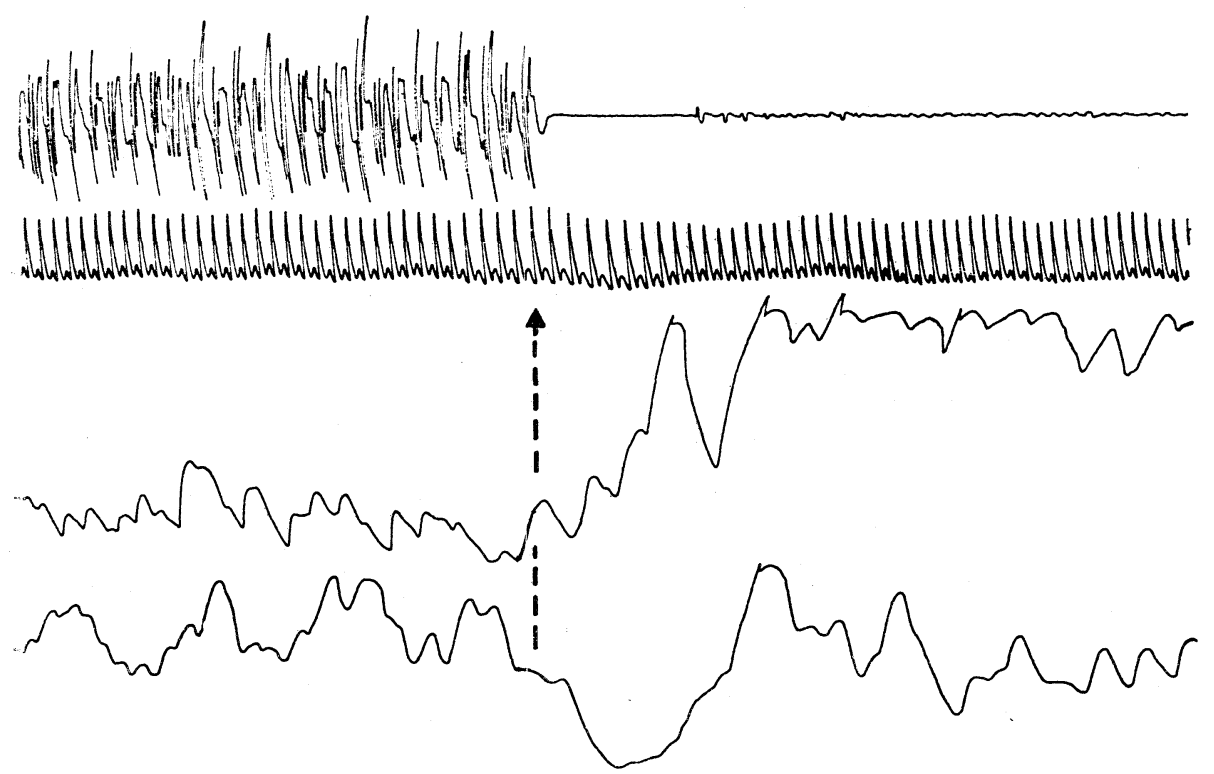

FIG. 9. Changes in cardiac sympathetic and parasympathetic activities occurring during the abrupt cessation of a self-sustained cortical activity episode. The records from the top downwards correspond respectively to the activity of anterior left sigmoid gyrus, systemic blood pressure, left cardiac vagus and sympathetic nerves integrated activity. The arrow shows the sudden ending of a cortical activity episode. At this moment the vagal activity increased and the sympathetic discharge was inhibited.

ship between the magnitude and temporal course of the cortical self-sustained activity and the magnitude of the increase in vagal discharge only at the beginning of the cortical episode, since approximately 20 to 30 seconds later the vagal discharge started to diminish without reaching the basal magnitude. When the self-sustained cortical activity entered into a clonic stage, the vagal response usually followed also this type of rhythmic discharge (FIGS. 2C and 5C). 2) In other experiments the initial increase vanished earlier and the control discharge reappeared in spite of the presence of a great self-sustained cortical activity. 3) A brief initial increase was followed by a reduction in activity. The latter was so great that the vagal action potentials were smaller than those recorded before the self-sustained activity (FIG. 5D). 4) Only a decrease in the vagal activity was observed during the whole period of self-sustained activity (FIG. 3).

Two types of modifications in vagal activity were recorded after the abrupt cessation of the self-sustained cortical response. a) An increase in vagal activity which lasted a short time and occurred simultaneously with the cortical silent period that usually followed an episode of self-sustained cortical activity (FIGS. 4C and 10). b) An initial reduction in the amplitude 

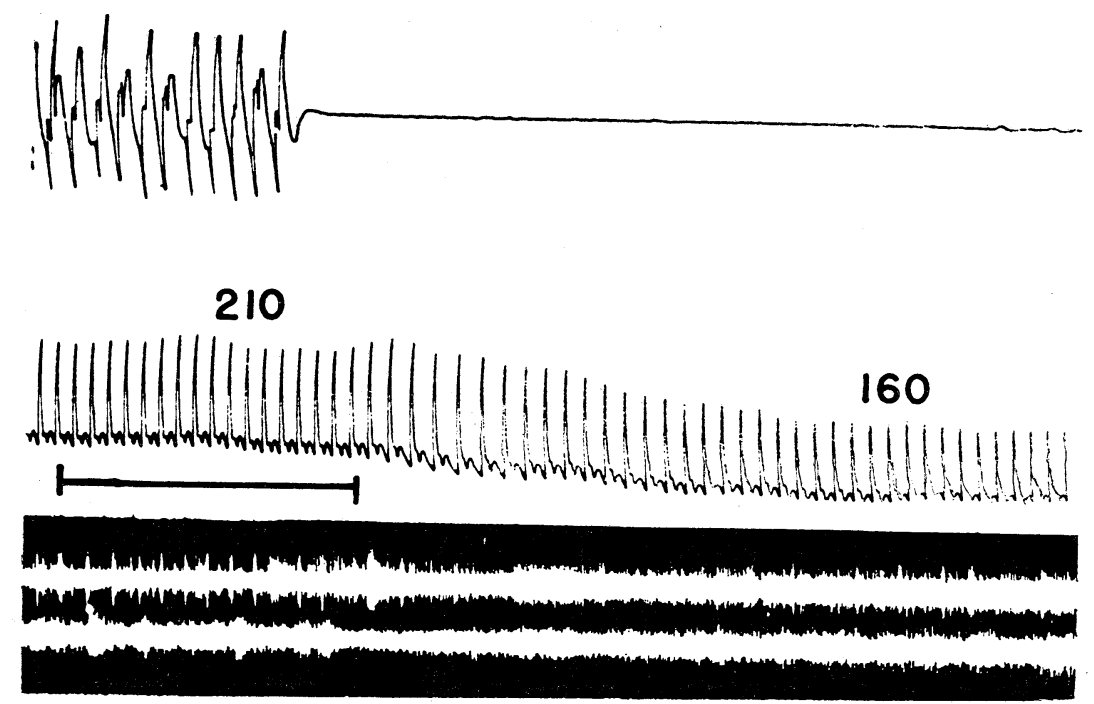

FIG. 10. The same phenomenon illustrated in FIG. 9. Note that a sudden diminution in heart rate (second trace) occurred during the increase in vagal discharge (third trace) concomitant with the sympathetic inhibition (lowest trace). In this case the hypertension produced by the self-sustained cortical episode disappeared in a short time after the cessation of the cortical activity (from 210 to $160 \mathrm{mmHg}$ ). Calibration $5 \mathrm{sec}$.

and frequency of vagal action potentials followed by a sudden increase (FIGS. $2 \mathrm{C}$ and $5 \mathrm{E})$. These two patterns of vagal responses were coincident with the abolition of the sympathetic activity mentioned in the corresponding section of this paper. The sudden increase in vagal response, which appeared during the cortical silent period, varied its magnitude according to the level of systemic blood pressure. At a high blood pressure $(200-220 \mathrm{mmHg})$ the vagal volley was greater than that elicited during a discrete hypertension. At a high blood pressure the cessation of the self-sustained cortical response was accompanied by a considerable reduction in heart rate, which occurred simultaneously with the sudden increase in vagal activity. This bradycardia lasted as long as the increment in vagal activity and coincided with the reduction or abolition of the sympathetic discharge (FIG. 10).

\section{DISCUSSION}

The methods.-The majority of the investigators, which postulate a specificity of cardiovascular representation in the cortex, based their assumptions on responses obtained by the stimulation of certain cortical areas. In general, these investigators did not take simultaneous recordings from the stimulated cortical region and from some other convolutions that could get activated indirectly. 
FRANçOIS FRANK (1887) and BARD (1929) considering this problem suggested that some of the effects produced by cortical stimulation could be due to the activation of areas adjacent to the stimulated region or to the activation of some subcortical structures located quite far from the area in question. Experimental findings (MORIN et al., 1953) point towards the same conclusion. Our observations also indicate that in order to attribute a response to the activation of a certain region, it is necessary to record not only from the stimulated area, but from some other functionally related areas. This becomes more important when the experiments are performed under light anaesthesia or on unanaesthetized animals. In these cases even weak and localized electrical stimuli are able to evoke a self-sustained cortical activity, which often propagates to other cerebral areas. The activity of these latter areas may produce the changes that are attributed to the stimulated region.

It is known that anaesthetics of different types diminish importantly the activity of the cerebral cortex. In our experiments (see AlANís et al., 1965) one seventh of the surgical anaesthetic dose considerably depressed the cortical activity. In other experiments the anaesthetic produced an inversion of the responses elicited by the electrical stimulation of the same point in the cortex. This effect has also been reported by Howell et al. (1900). It is important therefore to avoid, as far as possible, the use of anaesthetics when studying. the influence of cortical structures on cardiovascular functions. In the paralyzed animal reflex activity as well as a self-sustained cortical responses can be evoked easily.

The paralyzed dog receives a rich afferent bombardment coming from different peripheral receptors which contributes actively to the integration of the cardiovascular functions. This fact has to be considered for analyzing the influence of pressoreceptors on the autonomic nervous system activity, specially during the production of cortical self-sustained response.

The semiquantitative measurement of the changes in pupillary size permitted to compare the magnitude of increments in the same animal.

The results.-Since the classical studies of ScHIFF (1874), DANILEWSKY (1875) and BocheFontAINE (1875) it has been accepted that the activation of the sigmoid gyri produces a rise in systemic blood pressure. These results have been widely confirmed by other investigators which have used dogs, cats and monkeys (Dusser de Barenne, 1924; Mettler, 1936; Hoff et al., 1936, 1940, 1951a and 1951b; BAILey et al., 1940; Hsu et al., 1942 ; Delgado, 1960 ; ANAND et al., 1956 ; LANGFORD, 1957, etc.). In a previous paper (AlANIS. et al., 1965) we have analyzed the influences of the self-sustained cortical activity on different effectors innervated by sympathetic and parasympathetic. fibers. Some of the changes reported in the above mentioned paper were not satisfactorily explained probably due to the intervention of sympathetic and parasympathetic actions, which have different magnitude and temporal course. 
Although the magnitude of the changes observed in the effectors should be the resultant of these two opposite actions (Rosenbluth, 1934) we wanted to correlate the electrical activity of these two types of nerve fibers with the self-sustained cortical response of the sigmoid gyri.

Evidence which shows the preferential influence of the sigmoid gyri activity on the sympathetic nerve discharge. The following results indicate that the direct or indirect activation of sigmoid gyri produces preferentially an increase in the activity of the sympathetic nervous system.

a) During the application of weak stimuli, which did not produce a selfsustained cortical response, it was possible to see an increase in sympathetic activity which lasted only as long as the stimulating period. This effect appeared together with a rise in systemic blood pressure, an increase in heart rate and a dilatation of the pupils. If the weak stimuli were applied to other cerebral areas (anterior portion of lateralis and ectolateralis gyri) no changes were recorded (AlANis et al., 1965). In some experiments, when stimulating the anterior sigmoid gyrus with extremely weak stimuli, only an increase in the size of pupils was observed. This fact suggests that the threshold for the pupillary response is low in the sigmoid (ALANís et al., 1965) as well as in the proreus and in the middle and inferior frontalis gyri 〈Hoff, 1936).

b) When the electrical stimuli were strong enough for evoking a selfsustained cortical activity, the above mentioned effects were of a greater magnitude and lasted as long as the self-sustained cortical response from the sigmoid gyri. The magnitude and duration of the increase in sympathetic activity kept a direct relationship with the magnitude and duration of the self-sustained episode of the sigmoid convolutions (FIGs. 1, 2, 6 and 7).

It is important to point out that the relationship between cortical and sympathetic activities was seen only during the occurrence of the self-sustained cortical event. Before and after this cortical episode no correlation could be observed between them. This would suggest that during the self-sustained cortical response a profuse bombardment from the sigmoid gyri would act on subcortical pathways and bulbar neuronal groups controlling their discharge. Under normal circumstances, the impulses coming from different cortical layers or neuronal groups enter in activity asynchronously and probably are insufficient to produce important changes in any of the "sympathetic integrating centers", wherever located. In other words, during the self-sustained cortical activity the "pace-maker" for the sympathetic discharge has been displaced from the brain stem to the cortex.

c) The sympathetic discharges followed the synchronized responses of the sigmoid gyri in one-to-one manner during the clonic activity of a selfsustained cortical episode. This is an additional support for our suggestion 
that the "pace-maker" for sympathetic activity is temporarily located at a cortical level. A similar observation is illustrated in FIG. 7 in which a spontaneous short cortical silent period appeared during the course of the self-sustained cortical activity. The abolition of sympathetic discharge was strictly simultaneous with this cortical silent period.

One of the advantages of recording from sympathetic fibers, instead of doing it from the effectors innervated by this system, is evidenced by the fact that the clonic manifestations of the cortex cannot be detected by the changes in systemic blood pressure or heart rate. Although the clonic discharge was generalized to whole sympathetic system, the rhythmic changes caused by the clonic activity are probably masked by the large inertia of the effectors.

d) The increase in systemic blood pressure, heart rate, pupillary size, as well as the increment in electrical activity of sympathetic cardiac nerves, elicited by the self-sustained cortical activity, ceased in the majority of the experiments, in an abrupt manner and simultaneously with the sudden ending of the self-sustained cortical response. The cessation of the increase in sympathetic activity could be explained in terms of the abolition of cortical activity, but the significant diminution or inhibition of the sympathetic discharge, which accompany the cortical silent period (FIGs. $1 \mathrm{C}$ and $2 \mathrm{C}$ ) cannot be explained satisfactorily. Although the electrical activity from the sigmoid gyri was obviously inhibited, the temporal course of the cortical and sympathetic inhibition was not parallel. The former being longer than the latter i. e., the control pattern of sympathetic discharge reappeared at the time at which the cerebral cortex was still inhibited (FIGs. $1 \mathrm{D}$ and $2 \mathrm{C}$ ). This reduction in sympathetic activity appeared regardless of changes in systemic blood pressure, since it was observed in those experiments in which the rise in blood pressure produced by the self-sustained activity, was prevented. This would indicate that the afferent impulses, specially those coming from different presso-receptor areas, are not playing an important rôle in the inhibition of the sympathetic discharge.

It is known (BRONK et al., 1934 ; Downing et al., 1963 ; Alanís et al., 1963) that an abrupt hypertension inhibits the sympathetic tonic discharge. It is pertinent to emphasize that the increase in sympathetic activity, evoked by the self-sustained cortical response, took place in spite of a rise in blood pressure. In this case the presso-receptor inhibitory effect was probably overcome by the intensive activation of cortical origin.

e) The stimulation of other cerebral areas different from the sigmoid gyri did not produce by itself an increase in sympathetic activity (FIG. 8B) which augmented only when the response of the former cerebral structures spread to the sigmoid regions (FIG. $8 \mathrm{C}$ ). The preponderance of these cortical regions is revealed by the fact that even the marked and long-lasting sym- 
pathetic inhibition observed after hypothalamic stimulation (PITTs et al., 1941) is overcome by the activity of the sigmoid gyri.

Other results pointing towards the same conclusions are those reported by HofF et al., (1936) who applying local anaesthesia on the sigmoid gyrus or isolating this region by undercutting, eliminated the pressor response, the acceleration in heart rate and the dilatation of the pupils, all produced by the electrical stimulation of this cortical region.

Modifications in the vagal discharge which appeared during and after a selfsustained cortical activity. Although we have grouped the changes of the electrical activity of vagal cardiac fibers in four different patterns, it was not easy to ascertain the experimental conditions which determined each one of these responses. Among the numerous variables, which complicate the analysis of this problem, the rich variety of fibers that form the cardiac vagus nerve should be mentioned. It is known for example, that baro-receptor stimulation enhances the activity of some vagal fibers (IRIUCHIJIMA et. al., 1963) and inhibits the discharge of other types of fibers (JEWETT, 1964). In fact, GREEN (1959) has described three different types of vagal fibers in the cat, while JEWETT (1964) reported in the same animal seven groups with different characteristics.

During the cortical self-sustained response, the influence exerted by peripheral receptors of different types, seems not to be relevant for vagal integration. This is evidenced by the fact that during the initial part of the self-sustained cortical activity the frequency and amplitude of vagal discharge increased considerably (FIGS. 2B and 5B) independently of the level in systemic blood pressure and even in the cases in which this was kept constant. If peripheral receptors would contribute to some extent to the integration of vagal activity, their influence would be minimized by the intensive bombardment coming down from the cortical regions. We have no satisfactory explanation for the considerable reduction in vagal activity which appeared in some experiments during the whole self-sustained activity episode (FIG. 3B, $\mathrm{C}, \mathrm{D})$ and in others during the second part of the self-sustained cortical activity (FIG. 5D).

The pattern of vagal discharge changed in a sudden manner when the self-sustained cortical activity abruptly ceased. These changes in the pattern of discharge seem to depend on the systemic blood pressure level, since no modifications were observed when the systemic blood pressure was kept low and constant $(100-120 \mathrm{mmHg})$ during the experiment.

The two types of modifications in vagal activity, which occur after the abrupt cessation of the self-sustained cortical response are simultaneous with the abolition or inhibition of sympathetic activity (FIGS. 2C, 4C, 9 and 10).

The magnitude of the diminution in heart rate observed during the ces- 
sation of the cortical activity (FIGs. 9 and 10) was preponderably determined by the magnitude of the sympathetic inhibition, which may be accompanied by an increase in vagal response. The latter could be small, but due to the occurrence of the sympathetic inhibition, a greater bradycardia was observed. These results confirm that there may be a reciprocity between sympathetic and parasympathetic effects. We should not expect however that an inhibition of sympathetic activity necessarily will cause an increase in vagal response. In fact, in our experiments it was possible to observe a bradycardia without an increase in vagal discharge when the sympathetic discharge was strongly inhibited. In other series of experiments (AlANís, 1962) we were able to show that a bradycardia can be elicited after cardiac vagotomy and this effect disappeared only when sympathetic nerves were severed. In other words, as RosEnBLUETH et al. (1934) pointed out, the effects of simultaneous "excitation of accelerator and decelerator nerves are the "resultant" of the two influences. The two effects according to Rosenblueth occur independently, as if each set of nerves was acting alone.

\section{SUMMARY}

1. On paralyzed dogs the electrical activity of the left sympathetic and parasympathetic cardiac nerves was recorded simultaneously with the activity of the cerebral cortex (sigmoid gyri).

2. The electrical activity of the sympathetic postganglionic fibers (superior cervical and "stellate" ganglia) increased when the anterior or posterior sigmoid gyri were activated. When a self-sustained activity was evoked in these areas the increase in sympathetic activity lasted as long as the cerebral self-sustained episode (FIGS. 1 and 2). The magnitude of the increase in the sympathetic discharge was in general parallel to the increase in the activity of the anterior sigmoid gyrus (FIGs. 6 and 7).

3. The self-sustained cortical response, evoked by the stimulation of convolutions other than the sigmoid, did not produce by itself changes in either the activity of sympathetic cardiac nerves, the systemic blood pressure, the heart rate or in the pupillary size (FIGS. 8B). Such modifications appeared only when the self-sustained activity spread from the stimulated area to the sigmoid gyri (FIG. 8C).

4. The pattern of the sympathetic activity of the cardiac nerves showed two types of responses (tonic and clonic) which coincided with the tonicclonic stages recorded from the anterior sigmoid gyrus (FIGS. 3 and 5). The self-sustained activity ended suddenly and at this moment the sympathetic discharge diminished or even was abolished (FIGS. 1, 2, 3, 5 and 9). This reduction in the sympathetic discharge appeared simultaneously with the cessation of self-sustained activity and lasted 2 to 13 seconds. 
5. The above mentioned effects were observed after bilateral vagotomy and also when the changes in systemic blood pressure, produced by the cortical self-sustained activity, were minimized by means of a compensator system. 6. The self-sustained cortical activity of the sigmoid gyri produced during the initial part an increase in the vagal discharge (FIGS. 4 and 5) which was in general followed by a gradual reduction; at the final part of the self-sustained episode the reduction was so evident that the vagal discharges were abolished.

7. Simultaneously with the abrupt cessation of the self-sustained activity and during the sympathetic inhibition an increase of the vagal activity was observed (FIGS. 4, 9 and 10). The cardiovascular changes observed during the self-sustained cortical activity are discussed considering the relationships between the magnitude and temporal course of sympathetic and parasympathetic efferent discharge.

This work was partially supported by a grant given by “The Mary Street Jenkins Foundation" to the Instituto Nacional de Cardiologìa.

\section{REFERENCES}

1) Alanìs, J., López, E. And Rosas, O. Changes in dog's coronary circulation by hypothalamic stimulation. Arch. Cardiol. Méx., 32: 743, 1962.

2) Alanìs, J. And Mascher, D. Cambios de la circulación coronaria provocados por la actividad refleja. Arch. Cardiol. Méx., 33 : 587, 1963.

3) Alanis, J., Mascher, D. And Miyamoto, J. La influencia de la actividad autosostenida de la certeza cerebral sobre la presión arterial y el gasto coronario del corazón del perro. Arch. Cardiol. Méx., 35: 11, 1965.

4) Anand, B.K. And DuA, S. Circulatory and respiratory changes induced by electrical stimulation of lymbic system (visceral brain). J. Neurophysiol., 19:393, 1956.

5) BARd, P. The central representation of the sympathetic system. A.M.A. Arch. Neurol. Psychiat., 22 : 230, 1929.

6) Bailey, P. AND Sweet, W.H. Effects on respiration, blood pressure and gastric motility of stimulation of orbital surface of frontal lobe. J. Neurophysiol., $3: 276$, 1940.

7) Bochefontaine, L. T. Contribution, à l'études des effects produits par l'excitation électrique du cerveau. C.R. Soc. Biol., $27: 324,1875$.

8) Bronk, D. W., Ferguson, L. K. and Solandt, D. Y. The discharge of sympathetic impulses from the stellate ganglion and its relation to cardiac reflexes. Amer. J. Physiol., 109 : 15P, 1934.

9) Crouch, R. L. And Thompson, K. Autonomic responses elicited by stimulating the frontal region of cerebral cortex of the cat. Anat. Record, 64:(Suppl.3), 11, 1936.

10) Danilewsky, B. Experimentelle Beitraege zur Physiologie des Gehirns. Pflïg. Arch., $11: 128,1875$.

11) Delgado, J.M.R. Circulatory effects of cortical stimulation. Physiol. Revs., 40 : (Suppl. 4), 146, 1960. 
12) Downing, E. And Siegel, J.H. Baroreceptor and chemoreceptor influences on sympathetic discharge to the heart. Am. J. Physiol., 204: 471, 1963.

13) Dusser de Barenne, J. G. and Kleinknecht, F. Ueber den Einfluss der Reizung der Grasshirnrinde auf den allgemeinen arteriellen Blutdruk. Z. Biol., 82 : 13, 1924.

14) François-Frank, C. E. Leçons sur les fonctions motrices du cerveau (réactions volontaires et organiques) et sur l'épilepsie cérébrale. Paris, O. Doin, 1887.

15) Green, J.H. Cardiac vagal efferent activity in the cat. J. Physiol., 149:47P, 1959.

16) Hoff, E. C. And Green, H. D. Cardiovascular reactions induced by electrical stimulation of the cerebral cortex. Am. J. Physiol., 117: 411, 1936.

17) Hoff, E. C. The influence of the cerebral cortex on visceral activity, with special reference to the heart and circulation--a review. London Hosp. Gaz., $44: 45$, 1940.

18) Hoff, E. C., Langford, H. G., Vester, J. W., Beckner, W. W. and Thomas, P.R. Strychnine facilitation of pressor responses evoked from cerebral cortex. $J$. Neurophysiol., 14 : 167, 1951a.

19) Hoff, E. C., Kell, J. F. Jr., Hastings, N., Sholes, D. M. and Gray, E. M. Vasomotor, cellular and functional changes produced in kidney by brain stimulation. J. Neurophysiol., 14 : 317, 1951b.

20) Howell, W. H. And Austin, M.F. The effect of stimulating various portions of the cortex cerebri, caudate nucleus and dura mater upon blood pressure. Am. J. Physiol., 3 : 22P, 1900.

21) Hsu, S., Hwang, K. And Chu, H. N. A study of the cardiovascular changes induced by stimulation of the motor cortex in dogs. Am. J. Physiol., 137: 468, 1942.

22) Iriuchijima, J. And Kumada, M. Activity of single vagal fibers efferent to the heart. Jap. J. Physiol., $14:$ 479, 1964.

23) Jewett, D.L. Activity of single efferent fibers in the cervical vagus nerve of the dog, with special reference to possible cardio-inhibitory fibers. J. Physiol., $175: 321,1964$.

24) KaAda, B. R., Pribram, K. H. and Epstein, J.A. Respiratory and vascular responses in monkeys from temporal pole, insula, orbital surface and cingulate gyrus. A preliminary report. J. Neurophysiol., 12:347, 1949.

25) Langford, H. G., Patterson, J. L. Jr. and Porter, R. R. Dynamics of the circulation in acute hypertension produced by stimulation of the cerebral cortex. Circulation Res., 5 : 268, 1957.

26) Morin, G., Corriol, J. ET Zwirn, P. Signification de l'hypertension artérielle provoquée par la stimulation électrique du gyrus sigmoide. J. Physiol. (Paris) $45: 202,1953$.

27) Mettler, C.C., Hamilton, W. F., Allen, L., Woodbury, R. A. and Mettler, F. A. Influence of the cerebral cortex upon the cardiovascular system. Anat. Record, 64: (Suppl. 3) 33, 1936.

28) Pitts, R.F., Larrabee, M. G. And Bronk, D.W. An analysis of hypothalamic cardiovascular control. Am. J. Physiol., 134: 359, 1941.

29) Rosenblueth, A. And Simeone, F. A. The interrelations of vagal and accelerator effects on the cardiac rate. Amer. J. Physiol., 110: 42, 1934.

30) Rosenblueth, A. and Cannon, W. B. Cortical responses to electric stimulation. Amer. J. Physiol., 135: 690, 1942.

31) Schif, M. Untersuchungen ueber die motorischen Funktionen des Grosshirns. Arch. exp. Path. 3 : 171, 1875 (transl. by F. Borel from Lezione sopra il systema nervoso encephalico. Florence, 1874). 